\title{
Filigrane
}

Écoutes psychanalytiques

\section{Mouvements : les entretiens préliminaires avec les « patients difficiles ». Entre résurgence du traumatique et attente croyante}

\section{Louis Pinard}

Volume 27, numéro 2, 2018

La consultation psychanalytique aujourd'hui, entre héritages et remaniements

URI : https://id.erudit.org/iderudit/1055751ar

DOI : https://doi.org/10.7202/1055751ar

Aller au sommaire du numéro

Éditeur(s)

Revue Santé mentale au Québec

ISSN

1192-1412 (imprimé)

1911-4656 (numérique)

Découvrir la revue

Citer cet article

Pinard, L. (2018). Mouvements : les entretiens préliminaires avec les " patients difficiles ». Entre résurgence du traumatique et attente croyante. Filigrane,

27(2), 81-90. https://doi.org/10.7202/1055751ar
Résumé de l'article

L'article se centre sur plusieurs des aspects relationnels et métapsychologiques particulièrement mobilisés lors des entretiens préliminaires avec les patients que l'auteur qualifie de " difficiles ». On montrera que la compréhension qu'on peut tirer d'enjeux économiques et narcissiques au coeur des problématiques psychiques représentées par ces patients permet de mieux établir l'indication du dispositif thérapeutique qui sera le plus approprié dans leurs cas, et facilite l'inscription de leur demande d'aide. Seront également évoqués les mérites, en termes de compréhension clinique, des rencontres évaluatives tenues en présence d'un groupe d'analystes. 


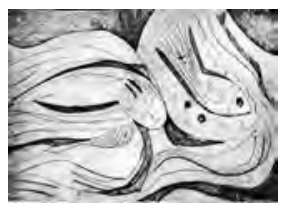

\title{
Mouvements: les entretiens préliminaires avec les "patients difficiles ». Entre résurgence du traumatique et attente croyante
}

\author{
Louis Pinard
}

\begin{abstract}
Résumé: L'article se centre sur plusieurs des aspects relationnels et métapsychologiques particulièrement mobilisés lors des entretiens préliminaires avec les patients que l'auteur qualifie de «difficiles». On montrera que la compréhension qu'on peut tirer d'enjeux économiques et narcissiques au cœur des problématiques psychiques représentées par ces patients permet de mieux établir l'indication du dispositif thérapeutique qui sera le plus approprié dans leurs cas, et facilite l'inscription de leur demande d'aide. Seront également évoqués les mérites, en termes de compréhension clinique, des rencontres évaluatives tenues en présence d'un groupe d'analystes.
\end{abstract}

Mots clés: entretiens préliminaires; patients «difficiles»; trauma; asymétrie de l'écoute; institution clinique.

Abstract: This paper focuses on several relational and metapsychological aspects particularly involved in the preliminary interviews with patients described as "difficult". It will be shown that the understanding of economic and narcissistic issues at the heart of these patients' psychological problems makes it possible to establish an appropriate therapeutic setting. It also facilitates the framing of their request for help. The heuristic contribution of an evaluative process held in the presence of a group of analysts will also be mentioned.

Keywords: preliminary interviews; "difficult" patients; trauma; asymmetry of listening; clinical institution.

Toute rencontre est un risque; à la première minute, aux premiers mots échangés, l'histoire, déjà, est en marche.

Raphaëlle Billetdoux, Mes nuits sont plus belles que vos jours

a clinique psychanalytique contemporaine s'est largement ouverte aux demandes des nombreux patients dont les manifestations de souffrance 
psychique s'inscrivent dans le registre non névrotique ou, pour le circonscrire rapidement, du narcissisme blessé, souvent gravement. Ce sont des troubles de la contenance et de l'impulsivité, des pathologies identitaires, états-limites, addictions, atteintes psychosomatiques, parfois des cas avérés de psychose, autant de symptomatologies hétérogènes, caractéristiques des patients que je désignerai dans ce texte par le terme générique de "patients difficiles». Au fondement de la configuration psychique de ces problématiques subjectives, on retrouve une carence d'inscription suffisamment souple et fonctionnelle de la figure du tiers, carence qui constitue l'obstacle parfois insurmontable à un travail psychanalytique "classique». Corrélativement à ce défaut d'inscription du tiers qui empêche l'accès à l'angoisse de castration et à la constitution d'un surmoi non exagérément inhibant, ces patients demeurent aux prises, à des degrés divers, avec la figure maternelle archaïque du temps de l'originaire, avec pour conséquences des problèmes topiques de différentiation moi/non-moi, de captation dans l'imaginaire, et de défaut de symbolisation. Chez de tels patients se trouve convoquée d'entrée de jeu et de manière marquée la problématique des origines, et notre attention doit se détourner «du Théâtre de l'Interdit avec CEdipe» au «Théâtre de l'Impossible marqué par Narcisse», pour reprendre la belle formule de Joyce McDougall (McDougall, 1982).

Au cours des dernières décennies, sous la poussée et l'intensité d'un pulsionnel délié dans la demande de ces patients qui la plupart du temps ne sont pas en mesure de tolérer le dispositif de la cure-type « divan-fauteuil ${ }^{1}$ » et la règle d'abstinence qui lui est inhérente, et grâce aux travaux pionniers sur le cadre de Pichon-Rivière et de Bleger en Argentine, puis d'Anzieu, Kaës et Roussillon en France, la référence à la cure-type comme prototype privilégié de «la situation analysante» (Donnet, 2005) s'est assouplie. Les exigences surmoïques qui ont longtemps condamné les analystes à privilégier un idéal du cadre analytique quasi sacralisé se sont progressivement adoucies, et parce que ce cadre témoin de fidélités et d'affiliations inhibantes aux figures tutélaires d'un passé psychanalytique personnel ou institutionnel n'a plus tenu la place d'un idéal absolu, de nouveaux dispositifs de soins ont pu être créés ${ }^{2}$.

Dans le rapport humain qui se développe au sein du couple analytique, atypique et non naturel (un des membres de la dyade acceptant de révéler à haute voix, à un autre le plus souvent silencieux, tout ce qui lui vient à l'esprit), l'asymétrie relationnelle constitutive de l'écoute et de la méthode a pour effet de provoquer un déséquilibre dans l'économie psychique du 
patient qui se sent plus ou moins happé dans un mouvement interne «en direction de l'autre», favorisant l'actualisation progressive de la névrose de transfert.

Les premiers entretiens n'échappent pas à cette dynamique, d'emblée marquée par ce que Michel de M'Uzan qualifie de «bouche de l'Inconscient» mobilisée par l'effet du silence de l'analyste. Même si lors de ces entretiens le clinicien n'est pas encore, à proprement parler, l'objet d'une névrose de transfert, il est, cependant, le réceptacle de transfert de fragments disparates et énigmatiques qui sont autant de déplacements, «d'affects flottants» (Ferenczi, 1909) - au sens premier de la conception du transfert, tel que l'entendait Freud dans L'esquisse.

Les patients les plus fragiles narcissiquement sont aussi les plus facilement déstabilisés par l'éprouvé d'angoisse qu'induisent ces premiers contacts et la mise en œuvre préliminaire de la méthode, qui invite d'emblée au déplacement. Ces patients ressentent, sans pouvoir les élaborer, la nature et la gravité de l'enjeu inconscient mobilisé qui semble entrer en résonance avec l'actuel hors-temps d'une première rencontre qui a pu être parfois catastrophique avec l'objet primaire.

Avec de tels patients chez qui la blessure est toujours vive, le thérapeute doit doser la frustration que la réserve impliquée par la règle d'abstinence peut provoquer, afin d'éviter que celle-ci ne soit vécue comme la répétition d'un trauma initial; au risque sinon de demeurer immun dans ces premiers contacts à la présence de l'autre, non affecté dans son corps et sourd dans son entendement, et de se réfugier dans l'esquive qui serait celle de voir dans ce patient un patient inanalysable. Dans le cas des "patients difficiles», le thérapeute est ainsi plus qu'à l'accoutumée interpellé par le traumatique et il essaiera de retracer l'histoire, ou à défaut, d'en construire une ébauche incertaine lorsque les matériaux en sont manquants. Agir ainsi est commandé par une quasi-impulsion contre-transférentielle, tant l'inconfort, voire l'angoisse convoquée, peuvent virtuellement troubler le clinicien.

Intimement lié au traumatique, le facteur économique constitue donc une donnée hyper-sensible dont on ne prend conscience parfois qu'en après-coup, d'où l'importance avec de nombreux patients de ne pas s'en tenir à un seul entretien lors du processus d'évaluation. À cet égard, les rencontres préliminaires dans un dispositif de groupe peuvent fournir un soutien inestimable au déchiffrage clinique d'éléments qui, à la suite des déplacements $^{3}$ que convoque chez le patient le processus d'évaluation, restent revêtus d'opacité. 
Nous avons ainsi pu constater avec profit dans le cadre du travail à la Clinique psychanalytique de Montréal (2007-2014), et aujourd'hui dans le Groupe psychanalytique du Mont-Royal (GPMR) dans les discussions de groupe qui font suite à un entretien préliminaire, ou lors de séances de supervision collective, comment, face à un même patient, un analyste participant en silence au processus a pu par exemple «sentir être déposés» en lui des affects de valence négative - avec toute une imagerie mentale à caractère violent qui s'ensuivait - tandis qu'un/une autre collègue faisait silencieusement l'expérience contre-transférentielle d'éprouver des fantasmes maternants tendres.

Clivages, affects flottants, contenus mal représentés, voire blancs de la pensée, sont autant d'énigmes que le travail groupal permet d'élaborer à la faveur, de la part du patient, de diffraction des transferts sur les diverses personnes présentes lors des entrevues préliminaires, permettant ainsi aux cliniciens d'avoir une première appréhension d'un prisme d'éléments béta plus ou moins désignifiés, et une amplification de leur résonance contre-transférentielle.

On sait qu'à la suite de ce qu'on a appelé le tournant des années vingt, après la Première Guerre et dans le contexte de drames personnels, Freud remanie sa théorie des pulsions et ouvre la voie à une re-fondation de sa construction métapsychologique en proposant une nouvelle dualité pulsionnelle qui oppose une force de liaison à une force de déliaison. Dans Le problème économique du masochisme (1924), Freud définit le masochisme originaire des tout premiers débuts de la vie comme la première intrication des deux pulsions antagonistes à travers leur excitation coextensive, un des destins de cette première intrication étant sa dérivation vers l'extérieur avec l'emprunt de trois chemins possibles, celui de la motricité, du sadisme et de la pulsion sexuelle de mort. Il s'agit dans la proposition freudienne d'un mécanisme purement endopsychique qui n'est aucunement lié à l'intervention de l'extérieur.

Dans une approche contemporaine qui fait davantage place au rôle de l'objet et à la transitionnalité, et en résonance avec des travaux de Denise Braunschweig (1971), Denys Ribas (2016) a développé la notion de «la mère intricante» qui permet un amalgame des deux pulsions, pulsion de vie et pulsion de mort, et fait tenir «ensemble le moi naissant».

Dans la lignée des travaux de Ferenczi, j'accorderai au traumatisme grave le rôle d'agent provocateur-amplificateur de la pulsion de déliaison, avec pour premier effet l'écrasement de la fonction de représentance et le 
maintien de la désintrication pulsionnelle et, pour effet corollaire, une insuffisance de possibilité de dérivation vers l'extérieur des quantas d'énergie non liés. Dans le propos central de son article fameux «L'enfant mal accueilli et sa pulsion de mort», on se souvient que Ferenczi montrait que c'est chez les patients ayant subi des traumas avant le développement du langage que l'impact sur la constitution de l'appareil psychique est le plus délétère.

Dans son texte «Les entretiens préliminaires et les mouvements d'ouverture», Piera Aulagnier a insisté sur l'importance de bien jauger lors des premières rencontres les risques de renforcement de Thanatos que peut induire le dispositif classique de l'analyse chez certains patients, jusqu'à mener parfois, soutiendra-t-elle, à une décompensation psychotique voire à un suicide. À cet égard, un dispositif clinique groupal d'évaluation d'une demande de support psychothérapique peut constituer une modification fertile du cadre habituel des entretiens préliminaires. Il aide à mieux évaluer les risques d'entreprendre un travail analytique classique avec un patient difficile, et peut parfois favoriser l'amorce d'une première intrication pulsionnelle prometteuse de la possibilité d'enclenchement d'un travail analytique (Lasvergnas, 2012).

De même, lorsque le traitement se déroule dans un centre de consultations, la réalité institutionnelle inscrite de facto dans le dispositif de la cure peut représenter pour certains sujets - qui autrement n'arriveraient pas à se le représenter - le socle transférentiel rassurant et "qui survit» sur lequel peuvent s'inscrire des fragments d'angoisses, voire de terreurs précoces dont le sens ne se dégagera que peu à peu. On peut en effet supposer que c'est parce qu'elle se soutient de son inscription dans le corps social - que le sujet sait être présent de tout temps, comme l'est le corps maternel pour l'infans - que la figure institutionnelle, par le fait de sa résilience, acquiert dans l'imaginaire les qualités de solidité et de durée, voire de pérennité. La présence de l'institution clinique peut ainsi agir pour le patient comme une des figurations possibles d'une stabilité de l'objet primaire et de la transitionnalité.

J'évoquerai en ce sens le cas d'un patient qui avait été très précocement et à plusieurs reprises séparé de sa mère en raison de chirurgies qu'elle avait dû subir, puis dont le développement fut grevé par le déclenchement chez sa mère d'une grave maladie qui allait la rendre de plus en plus affaiblie sur les plans cognitif et locomoteur. Cet homme qui se voyait au sortir de l'adolescence destiné à une vie d'intellectuel universitaire se retrouvait au mitan de la vie sans attaches et sans ressources financières stables. 
Lorsqu'il se présente en consultation à la CPM, ce patient issu d'une union tardive entre une mère francophone et un père anglophone, souffre d'un syndrome de fatigue chronique qui le garde inactif une bonne partie de la journée. Il est suivi pour cette condition par un médecin infectiologue depuis une dizaine d'années, sans grand effet curatif, malgré l'administration par voie parentérale d'une médication antivirale de façon récurrente.

En raison d'un noyau dépressif persistant qu'il n'arrive pas à élaborer avec la thérapeute qu'il consulte depuis quelques années, il s'adresse à la Clinique «pour avis» dit-il, et dans l'espoir également de relancer avec un nouveau thérapeute un travail psychothérapique qu’il juge stagnant "parce que sa thérapeute ne dit pas un mot». Nous découvrons alors qu'il est luimême difficile à interrompre, parlant sans discontinuer de son histoire infantile douloureuse, d'une voix si basse qu'il en est presque inaudible, et ne laissant aux deux analystes consultants aucune place pour intervenir. Il nous transmet la sensation qu'il est une sorte de dompteur de lions, que ma collègue et moi sommes alors pour lui. Orientation sexuelle mal définie nous dira-t-il, lien au père difficile depuis sa prime enfance, mais «brisé» vers l'âge de 12 ans parce que le jeune adolescent lisait «de mauvais livres».

Un peu paranoïde, il nous demande pourquoi nous souhaitons noter son adresse postale et tient à s'assurer qu'il n'est pas filmé durant le processus de consultation. Malgré sa tristesse mélancolique très palpable, et sa sexualité trouble, le mécanisme de défense massive que constitue son côté très contrôlant inquiète ma collègue. Nous jugeons tous deux qu'il s'agit d'un cas de traumatisme originaire lourd - «enfant mal accueilli» de par les absences précoces puis de la très grave maladie de la mère, menant peutêtre un combat silencieux contre une envie de mourir (Ferenczi, 1929, p. 78-79 $)^{4}$ - et nous sommes hésitants sur l'indication de traitement. Après discussion en séminaire élargi avec l'ensemble de l'équipe clinique, et grâce au relatif écart dans l'écoute contre-transférentielle prêtée à ce patient par les deux analystes consultants, il est décidé de lui offrir un traitement en face à face, une fois par semaine au début, avec un thérapeute masculin et bilingue, moi-même en l'occurrence, ce que le patient acceptera de manière ambivalente.

La thérapie, ardue, avec ce patient inaudible et souvent trop "épuisé» pour se rendre à ses séances, finit quand même par faire sens petit à petit. À mesure qu'il élabore timidement la relation à un père gardé à distance depuis toujours "parce qu'il ne (le) comprenait pas», le patient prend conscience d'une homosexualité passive qui l'angoisse beaucoup et dont il se défend 
dans le transfert avec moi. Demeure en même temps non abordée la question centrale à mes yeux de l'identification massive à la mère. Celle-ci pourtant est omniprésente: silencieusement en séance par le choix de la langue parlée et par le symptôme de fatigue chronique; et, hors séance, dans un transfert latéralisé en direction de la Clinique dont il contacte la directrice à plusieurs reprises, soit pour lui demander des conseils sur comment faire pour devenir psychanalyste; soit, pour s'assurer que ses craintes - non fondées et à caractère vaguement paranoïde - de perdre le soutien financier qui lui est offert pour son traitement ne se matérialisent pas.

Durant des séances de supervision de groupe, j'apprendrai que lors de ces contacts téléphoniques, et sans le demander formellement, il indique de façon implicite le fantasme de continuer la thérapie avec la personne de la directrice de la CPM, qu'il semble idéaliser d'emblée (il ne l'a jamais rencontrée), alors qu'il a tendance à me considérer avec un certain mépris dans le transfert.

Je pense reconnaitre dans ce double mouvement transférentiel clivé, entre dévalorisation manifeste de l'objet paternel et idéalisation apparente de l'objet maternel, l'un qui m'est adressé en face à face, et l'autre qui s'exprime latéralement et qui prend appui sur l'instance tierce de la Clinique, la réédition inconsciente d'un noyau crucial du scénario infantile du patient. Lorsque je l'aurai informé de ma désaffiliation de la CPM, et que je lui offrirai de continuer le processus avec lui aux mêmes conditions matérielles que celles qui prévalaient jusque-là, il décidera abruptement de ne pas poursuivre le traitement, sans qu'il soit possible de lui offrir un espace-temps minimum pour élaborer le mouvement d'angoisse qui suscitait chez lui son impulsion de rupture.

En rétrospective, je suis convaincu aujourd'hui qu'un premier travail essentiel concernant la figure du père dans son versant homo-érotique n'avait été possible que parce qu'un transfert maternel massif avait été défléchi sur la figure institutionnelle de la CPM projetée dans ce cas sur la figure de sa directrice. Cette part de transfert qui n'avait pu être analysée portant elle-même, toutefois, la part sombre et négative d'une mère malade et abandonnique.

La traversée des entretiens préliminaires avec les patients difficiles est un parcours miné. L'analyste l'aborde avec les outils à sa disposition que 
sont ses connaissances théoriques, son expérience clinique, mais surtout son désir-d'analyste et sa croyance convaincue en l'existence de l'inconscient. Pour sa part, le patient sera dans une disposition psychothérapique plus favorable lorsqu'il ressent en lui «l'attente croyante et pleine d'espoir» envers son «médecin», cette "force agissante» dans le processus de guérison, ainsi que l'écrit Freud dans un texte datant de 1890 (p. 162) 5 .

Toutefois, lorsque les premiers entretiens sont vécus par le patient comme une plongée de soi devant l'autre, massive, soudaine, impulsée du dedans, il incombe à l'analyste de penser cette plongée comme un mouvement de bascule, à l'image, dirait Pascal Quignard (2013, p. 42), dans l'expérience de la naissance du «corps qui tombe sur la terre neuve». Une «opération» physico-psychique imprimant chez le patient un mouvement pré-transférentiel énigmatique très condensé, voire inaudible, qui, du côté de l'analyste est davantage à ressentir qu'à comprendre. L'analyste doit dans de tels cas pouvoir, le premier, faire acte de croyance que du transfert a lieu, qu'il est là en puissance et prêt à se manifester, et doit s'ancrer, au moins temporairement, au matériel associatif que la rencontre génère en lui ${ }^{6}$.

Nous nous appuierons à ce propos sur la distinction «subtile» et précieuse que Catherine Chabert (2015, p. 18) introduit entre conviction et croyance. La conviction en analyse est le résultat, précise-t-elle, d'un long travail «produit ou effet» de la perlaboration. «Elle est le fruit de ce qui se construit dans le transfert ${ }^{7}$ » et se forge chez l'analyste par l'expérience des transferts répétés au fil du temps ${ }^{8}$. Par opposition, l'immédiateté d'adhésion que signe le mouvement de croyance «relèverait d'opérations plus massives, plus instantanées, davantage prises dans "l'affect et l'éprouvé suscités par l'objet auquel [la croyance] s'attache", susceptible qu'est celle-ci "de rejaillir [...] aux premiers moments de la rencontre avec un patient [... concernant] alors davantage l'essentiel moteur de la cure, c'est-à-dire le transfert, dont on ne peut jamais dire qu'il n'a pas lieu, même s'il reste sans effets, même et surtout s'il n'est ni reconnaissable, ni identifiable" (nous soulignons).

Vers la fin de son article "Le credo du psychanalyste ${ }^{9}$ » Green (1978) situe les fondements de la croyance en soi et en l'autre dans le rapport mèreenfant, en particulier au moment de l'échange de sourires qui permet à l'enfant de croire en la possibilité d'une réalisation de désir. À Winnicott qui disait que l'enfant se voit dans le regard de la mère, Green ajoute donc l'importance pour celui-ci de se voir «dans son sourire» (Green, 1978).

En ce sens, et de manière analogique, je soutiendrais que ce qui se joue lors des premiers entretiens fait écho aux premières rencontres mère-enfant 
lors de la genèse de la «con-fiance» mutuelle, en particulier dans la perception de regards échangés avec l'objet dans lequel l'enfant se voit sous valence de plaisir partagé

Et plus particulièrement, lors de la rencontre initiale avec des patients très fragiles, se trouve réactivé chez le thérapeute en réponse à la souffrance de l'autre, dans la proximité des corps en présence mutuellement perçus, ressentis, éprouvés, le complexe d'accueil d'un maternel primaire, ou encore le programme inscrit au cour de l'humain visant à secourir et à empêcher l'anéantissement: l'analyste occupant par son écoute la place du Nebenmensch -l'autre secourable, ce que fut sans conteste, dans la rencontre psychanalytique fondatrice, Fliess pour Freud, «l'être-humain-proche, le témoin, le voisin».

\author{
Louis Pinard \\ louis.pinard@mcgill.ca
}

\title{
Notes
}

1. Avec trois ou quatre séances hebdomadaires.

2. La pluralité de cadres cliniques remaniés, "sites dérivés» comme les nomme Baldacci (2006), sont ceux des psychothérapies de groupe, des thérapies de couple et familiales, du psychodrame psychanalytique, des suivis conjoints du patient en médecine avec utilisation de médications psychotropes, des traitements pluriels en institution, de l'artthérapie, de la musico-thérapie, etc., qui ont formalisé l'extension du cadre classique et introduit la figure d'un tiers «objectivé».

3. Au sens premier du transfert chez Freud, tel qu'évoqué plus haut.

4. On a en tête l'énoncé de Ferenczi indiquant «la probabilité du fait que des enfants accueillis avec rudesse et sans gentillesse meurent facilement et volontiers. Ou [qu']ils utilisent un des nombreux moyens organiques pour disparaître rapidement».

5. L'attente croyante est la préfiguration de la capacité au transfert que Freud n'a pas encore mis en lumière.

6. Ce qui n'empêche pas la prudence, car il faut se rappeler que tout demeure incertain et marqué par l'imaginaire, et que restera alors dans le travail de la cure, comme l'élabore essentiellement Donnet dans ses réflexions théoriques, de devoir passer de la séduction de l'analyste comme personne, à la séduction de la méthode.

7. Laurence Khan, citée par C. Chabert (2015).

8. Sans oublier l'expérience de l'analyse personnelle qui emporte plus que tout, chez l'analyste, sa conviction la plus intime en l'existence de l'inconscient.

9. Alors qu'il fait état d'une discussion tenue avec des scientifiques sur l'existence de l'inconscient lors d'un symposium interdisciplinaire sur la réalité.

\section{Références}

Baldacci, J.-L. (2006). La psychanalyse dans la cité. Dans R. Perron (dir.), Psychanalystes, qui êtes-vous? (p. 49-55). Paris: Inter-Éditions.

Braunschweig, D. (1971). Psychanalyse et réalité. À propos de la théorie et de la technique psychanalytique, Revue française de psychanalyse, 35 (5-6), 655-800. 
Chabert, C. (2015). Croire au transfert. Annuel de l'Association psychanalytique de France, $2015(1), 15-32$.

Donnet, J.-L., (2005). La situation analysante. Paris: Presses universitaires de France.

Ferenczi, S. (1929). L'enfant mal accueilli et sa pulsion de mort. Dans M. Balint et J Dupont (dir.), Psychanalyse IV, (p. 76-81), 1990.

Ferenczi, S. (1909). Transfert et introjection. Dans M. Balint et J. Dupont (dir.), Psychanalyse I, (p. 93-125), 1990.

Freud, S. (1924). Le problème économique du masochisme. Dans Euvres complètes, t. XVII. Paris: Presses universitaires de France, 1992.

Freud, S. (1890). Traitement psychique (traitement d'âme). Résultats, idées, problèmes, I, Paris: Presses universitaires de France, 1984.

Green, A. (1978). Le credo du psychanalyste. Incroyable mais vrai. Nouvelle revue de psychanalyse, $18,263-272$.

Lasvergnas, I. (2012). La clinique psychanalytique de Montréal: cinq ans d'existence. Un projet d'engagement social de la psychanalyse et un dispositif d'écoute original. Revue Filigrane, 21 (2), 55-70.

McDougall, J. (1982). Théâtres du Je (1ère éd.). Paris: Gallimard.

Quignard, P. (2013). L'origine de la danse. Paris: Galilée.

Ribas, D. (2016). Intrication et désintrication pulsionnelle dans la névrose et la naissance psychique: la mère intriquante. Revue canadienne de psychanalyse, 24 (1), 3-18. 